\title{
Use of Dexmedetomidine in Pediatric Patients for the Decrease of the Postoperative Delirium: Implementation of a Protocol Approved for Premedication

\author{
Patricia Catalán Escudero ${ }^{*}$, Adolfo Ramos Luengo ${ }^{2}$, Eva Delgado Encinas ${ }^{3}$ and \\ Ernesto Martínez García ${ }^{4}$
}

\begin{abstract}
${ }^{1}$ Anesthesiology, Reanimation and Pain Therapy Department, Niño Jesús University Children's Hospital, Madrid, Spain
${ }^{2}$ Anesthesiology, Reanimation and Pain Therapy Department, Hospital Universitario Severo Ochoa, Leganés, Madrid, Spain ${ }^{3}$ Surgical Block Nurses, Niño Jesús University Children's Hospital, Madrid, Spain

${ }^{4}$ Head of Anesthesiology, Reanimation and Pain Therapy Department, Niño Jesús University Children's Hospital, Madrid, Spain
\end{abstract}

*Corresponding author: Patricia Escudero Catalan, Anesthesiology, Reanimation and Pain Therapy Department of the Niño Jesús University Children's Hospital, Calle Nuestra Señora de Valverde 201 portal J $3^{\circ}$ A, Madrid, Spain, Tel: 0034649695919

\begin{abstract}
Background: Delirium is a very frequent alteration of postoperative behavior in children. Tonsillectomy surgery is related to severe sleep apnea and does not indicate benzodiazepine premedication. Non-premedication is directly related to the presence of negative behaviors. Dexmedetomidine is a safe option for children with obstructive sleep apnea (OSA) since it does not affect airway tone.

Aims: The main aim of this retrospective observational study is determining whether intranasal dexmedetomidine premedication decreases the incidence of preoperative anxiety, postoperative delirium, and the need for postoperative sedatives and opioids in children undergoing tonsillectomy.

Methods: 83 patients aged between 2 and 11 years were enrolled in a retrospective observational study in two groups according to receiving $1 \mu \mathrm{g} / \mathrm{kg}$ of intranasal dexmedetomidine premedication or not. Preoperative anxiety was assessed using the Yale Preoperative Anxiety Scale (YPAS). Postoperative Delirium was measured with the Pediatric Anesthesia Emergency Delirium (PAED) at 15, 30, and one hour after admission into the Postanesthetic Care Unit (PACU). Demographic data, intra-operative analgesics, post-operative opioids and sedatives administered, post-operative bleeding and perioperative respiratory adverse effects (PRAEs) were collected.
\end{abstract}

Results: Study groups were comparable. The incidence of preoperative anxiety was $57.89 \%$ in non-premedicated compared to $26.67 \%$ in the dexmedetomidine group ( $p=$ 0.008 ). The delirium at 30 minutes was $52.63 \%$ in non-premedicated compared to $28.89 \%$ in premedicated $(p=$ 0.048 ). Anxiety or delirium occurred at any time was $92.11 \%$ in non-premedicated compared to $68.89 \%$ in premedicated patients $(p=0.0194)$. No statistically significant differences were found in the need for intraoperative or postoperative opioids or postoperative sedatives, bleeding, or PRAEs. No cases of hypotension, bradycardia have occurred.

Conclusion: The incidence of preoperative anxiety and delirium in children without premedication is very high. Premedication with $1 \mu \mathrm{g} / \mathrm{kg}$ intranasal dexmedetomidine reduces preoperative anxiety and the incidence of anxiety and delirium at any time in the perioperative period.

\section{Keywords}

Dexmedetomidine, Premedication, Delirium, Children, Pediatric

\section{Introduction}

Delirium or agitation upon awakening is a very frequent alteration of postoperative behavior in children (up to $80 \%$ ), especially at school age and general anesthesia with sevoflurane. It is accompanied by an incre- 
ased risk of self-injury, bleeding from surgical bed dehiscence, and requires a higher dose of sedatives, which can delay discharge [1-3]. In addition, non-premedication has been related to preoperative anxiety in children of up to $65 \%$ and is directly related to the presence of negative behaviors such as postoperative delirium [4]. Anxiolysis before induction of anesthesia is, therefore, an important aspect of pediatric perioperative planning.

Benzodiazepines are the most widely used drugs as preoperative anxiolytic medication in pediatric patients [5]. Tonsillectomy surgery is related to postoperative delirium [1] but because of the risk of perioperative respiratory adverse events (PRAEs) in obstructive sleep apnea (OSA), benzodiazepines are not indicated as premedication in these patients.

Dexmedetomidine is a potent $\alpha 2$ adrenoreceptor agonist with analgesic effects without causing respiratory depression like opioids, so it may be safer. It also has sedative effects by inhibition of the locus coeruleus, reducing agitation, and sympatholytic effect by decreasing the release of norepinephrine in the sympathetic nerve endings. Dexmedetomidine is 8 times more powerful and more specific $(\alpha 2 / \alpha 1,1620 / 1)$ than clonidine $(\alpha 2 / \alpha 1,220 / 1)$ allowing it to have fewer side effects $[4,6,7]$.

Administration of Dexmedetomidine at the end of surgery or continuous infusion has better results in ter$\mathrm{ms}$ of preventing postoperative delirium than bolus propofol at the end of surgery or continuous intraoperative ketamine administration [8].

Currently, the United States Food and Drug Administration (FDA) approves sedation through intravenous bolus and continuous infusion up to $24 \mathrm{~h}$ in intubated adults, areas outside the intensive care unit (ICU) and the operating room environment. However, in Europe, it is indicated for sedation of adults in the ICU and non-intubated adult patients, before and/or during diagnostic or surgical procedures that require sedation. Although there is currently no worldwide approval for administration to the pediatric population in the operating room, the favorable pharmacological effects of dexmedetomidine combined with its limited adverse effect profile have facilitated its introduction into the perioperative environment and in children as compassionate use [9].

Dexmedetomidine, administered in dose even in higher than recommended, has been shown as a safe option for children with OSA since it does not affect airway patency and tone [9].

Nowadays, most studies of postoperative agitation in children have been performed with single or continuous intravenous doses of dexmedetomidine. Although the ability of dexmedetomidine to decrease preoperative anxiety has been studied, the data are limited in defining the dose and route of administration in premedication for the reduction of postoperative anxiety and postoperative pain [7].

It is hypothesized that intranasal dexmedetomidine premedication decreases the incidence of preoperative anxiety, postoperative delirium, and the need for postoperative sedatives and opioids. We have therefore designed a retrospective observational study with the main aim of determining whether this is achieved in children undergoing tonsillectomy \pm adenoidectomy. Incidence of adverse respiratory effects, bleeding or reintervention will also be evaluated.

\section{Materials and Methods}

Our study was designed to analyze the effect of dexmedetomidine premedication in children on the incidence of delirium and agitation after tonsillectomy \pm adenoidectomy. After obtaining approval of the Pediatric University Hospital Niño Jesús (Madrid) ethical committee, and written informed consent from parents or legal representatives, 83 patients were enrolled in a retrospective observational study following the STROBE statement.

\section{Participants}

Children with OSA aged between 2 and 11 years, scheduled for tonsillectomy surgery under general anesthesia using sevoflurane for induction and maintenance were included. Children who underwent adenoidectomy only, with total intravenous general anesthesia, a previous diagnosis of delirium, children who received intraoperative and/or postoperative dexmedetomidine or with contraindication for the use of dexmedetomidine were excluded.

Patients were included in one of the study groups according to receiving dexmedetomidine premedication or not: GROUP A (not premedicated) or GROUP B (Premedicated). The administration of benzodiazepines or opiates as a pre-medication was contraindicated in all patients included for OSA following the premedication hospital's protocol.

\section{Study procedure (Figure 1)}

Group A patients received $1 \mu \mathrm{g} / \mathrm{kg}$ of intranasal dexmedetomidine with atomizer 45 minutes before the intervention. Group B patients received no anxiolytic premedication.

Blood pressure (BP) and heart rate (HR) were measured before premedication and 30 minutes later. $A$ drop in BP or HR $>30 \%$ was considered a hemodynamic event.

Preoperative anxiety was assessed using the Yale Preoperative Anxiety Scale (YPAS) [10] by anesthetic staff trained on the scale.

During anesthesia, drug doses and programming parameters of different devices were standard.

After surgery, patients were transferred to the 


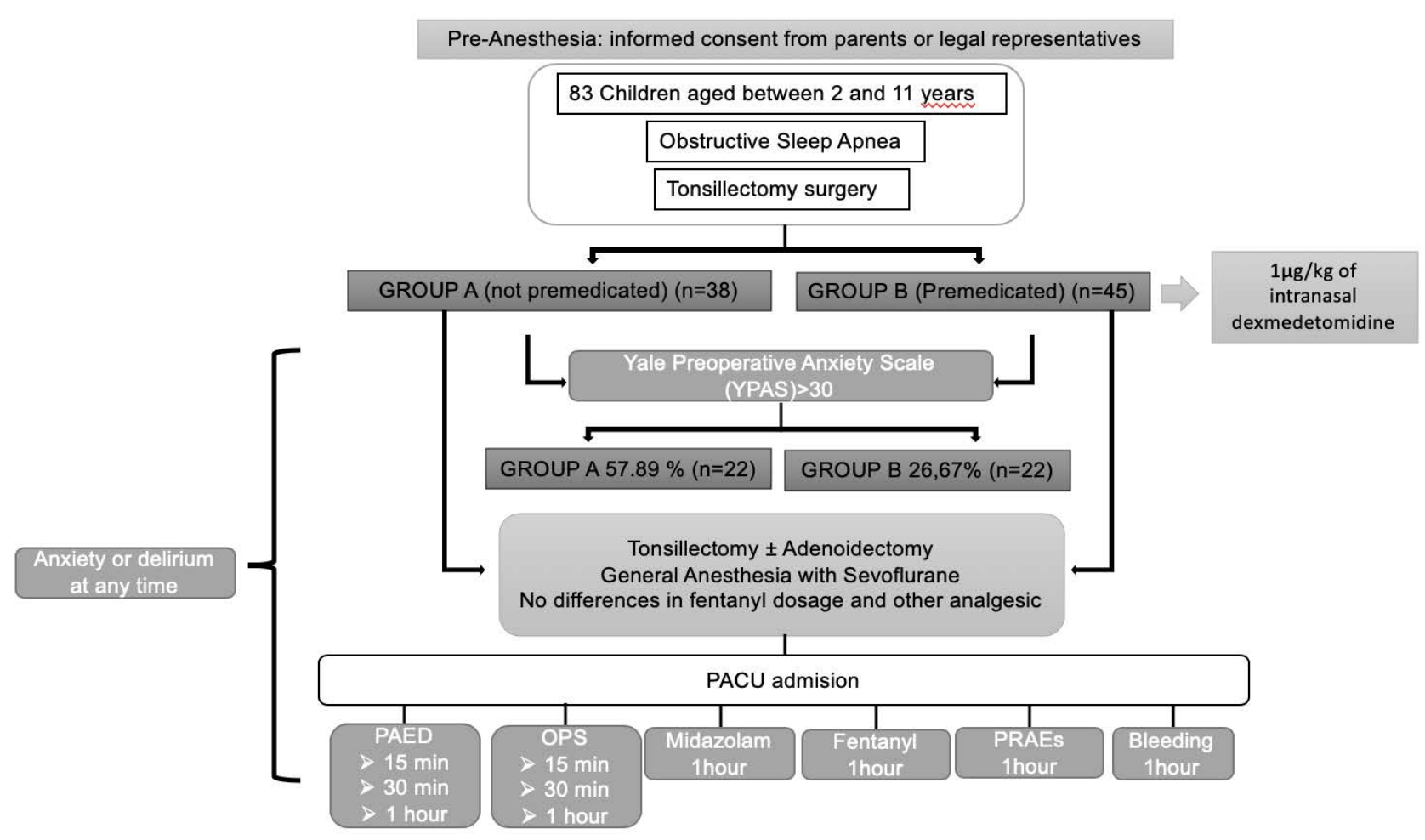

Figure 1: Study procedure.

Table 1: Demographic Data: Data are listed as "\% (value)" or as "median [interquartile range]". P-Values are by Mann-Whitney U for continuous data and by Chi-square for \% measures, or by Fisher's exact test when cell counts were less than 5 for qualitative data.

\begin{tabular}{|l|l|l|l|}
\hline & Dexmedetomidine (n= 45) & No premedication $(\mathbf{n}=38)$ & p value \\
\hline Age (years) & $3(2)$ & $4(2)$ & 0.619 \\
\hline Ratio Women/men & 0.6 & 0.71 & 0.412 \\
\hline Weight $(\mathrm{kg})$ & $17(4)$ & $17(6)$ & 0.409 \\
\hline ASA I & $4.44 \%(2)$ & $2.63 \%(1)$ & 0.897 \\
\hline ASA II & $91.11 \%$ & $92.11 \%$ & 0.897 \\
\hline ASA III & $4.44 \%(2)$ & $5.26 \%(2)$ & 0.897 \\
\hline Intraoperative Fentanyl $\mathrm{\mu g} / \mathrm{Kg}$ & $2.14(0.85)$ & $2.11(0.6)$ & 0.363 \\
\hline
\end{tabular}

post-anesthesia care unit (PACU) where they received analgesic treatment following our hospital's mild-moderate pain protocol.

Delirium on awakening from anesthesia was measured by the Pediatric Anesthesia Emergency Delirium (PAED) [11] at 15, 30 and 1 hour after PACU admission. A value of PAED $>10$ in any measurement was considered delirium and midazolam $0.5-1 \mathrm{mg} / \mathrm{kg}$ was administered.

Postoperative pain was assessed using an objective pain scale (OPS) [12] at 15,30 , and 1 hour after admission into the PACU. If rescue analgesia was required (OPS > 3), fentanyl $0.5 \mu \mathrm{g} / \mathrm{kg}$ was administered.

Demographic data (Table 1), premedication administered, intra-operative anesthetics and analgesics, post-operative opioids and sedatives administered, post-operative bleeding and PRAEs were collected.

\section{Statistical analysis}

Quantitative data were checked for normal distribu- tion by the method of Kolmogorov-Smirnov-Lilliefors. Differences between subgroups were analyzed by a Student's t-test or the Mann \& Whitney $U$ test depending on their distribution. Categorical data were analyzed by a Chi-square test with Yates's continuity correction. A $p$-value of 0.05 was considered significant.

Statistical analysis was performed using R $3.6 .1 \mathrm{bi}$ nary for MAC OS $\times$ 10.13.6.

Sample size calculation has been performed to obtain a power of $80 \%$ with an alpha error of $5 \%$ and using the data published by G Guler, et al. [13].

\section{Results}

Finally, 83 children were included. Study groups were comparable in terms of demographics and intraoperative fentanyl doses (Table 1).

The incidence of preoperative anxiety measured with the YPAS scale was $57.89 \%$ (22) in the non-preme- 


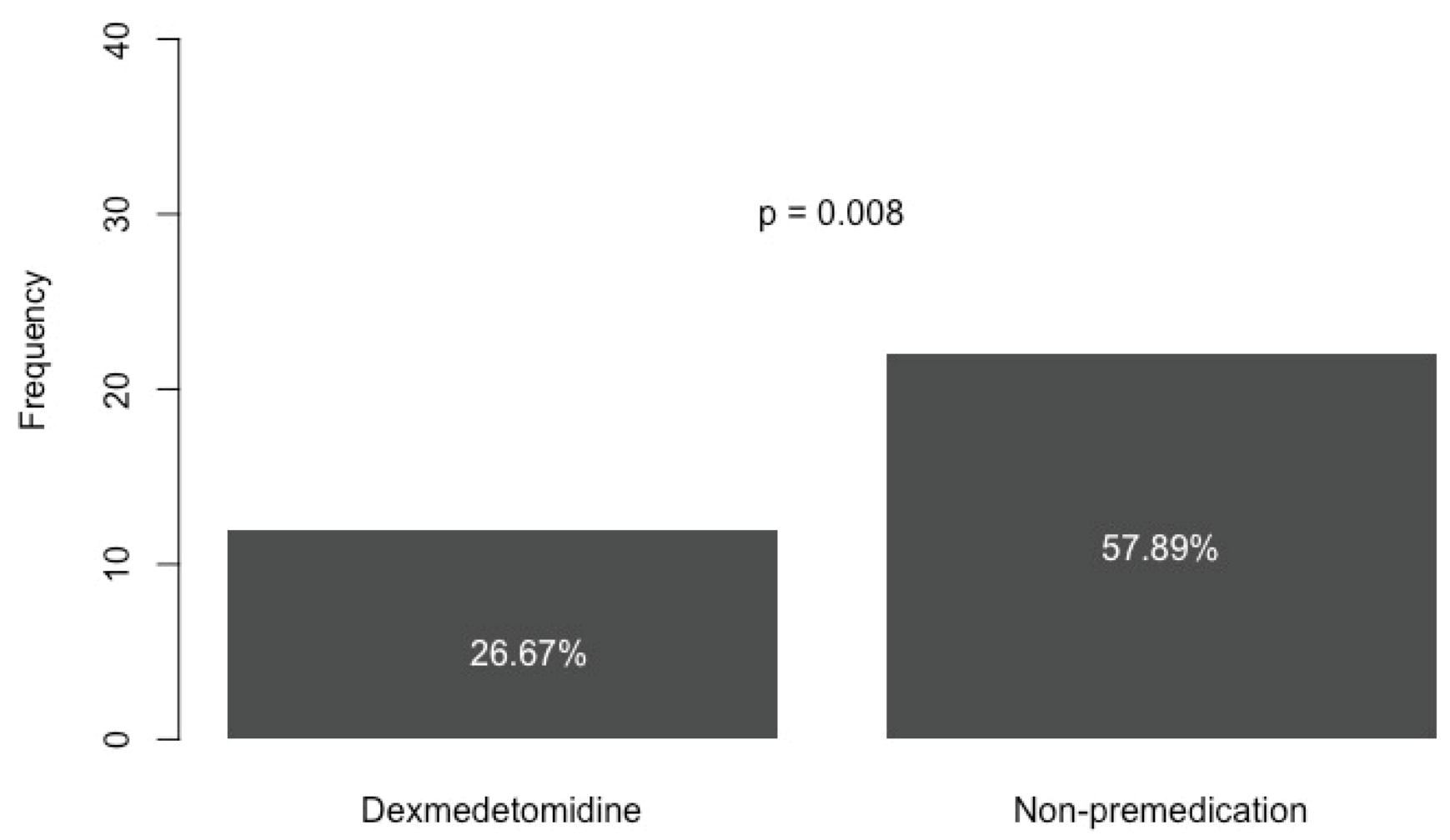

Figure 2: Preoperative anxiety.

dicated patients compared to $26.67 \%$ (12) in the dexmedetomidine premedicated patients $(p=0.008)$.

The delirium defined as a PAED > 10 at 15 minutes, was registered in the $63.16 \%$ of the non-premedicated children, and in the $42.22 \%(24)$ in the premedicated group ( $p=0.09$ ). At 30 minutes it was $52.63 \%(19)$ in non-premedicated patients compared to $28.89 \%$ (13) in premedicated patients $(p=0.048)$ and at one hour it was $10.53 \%$ (4) in premedicated patients compared to $22.22 \%(10)$ in premedicates $(p=0.26)$ (Figure 2).

Anxiety or delirium occurred at any time in the postoperative period of $92.11 \%$ (35) in non-premedicated patients compared to $68.89 \%$ (31) in premedicated patients $(p=0.0194)$.

No statistically significant differences were found between patients with or without dexmedetomidine in adverse respiratory events: bronchospasm (0\% vs. $2.63 \%(1) ; p=1)$, desaturation (15.56\% (7) vs. $18.42 \%$ $(7) ; p=0.958)$ or stridor $(4.44 \%$ (2) vs. $10.53 \%(4) ; p=$ $0.521)$.

No cases of hypotension, bradycardia, post-operative bleeding, or need for reoperation have occurred.

\section{Discussion}

Pre-operative anxiety in non-premedicated children is a serious issue that can occur in up to $65 \%$ and it is associated with negative behaviors such as nightmares, enuresis, night crying, and postoperative delirium [4]. In our study, we found a slightly lower frequency $(57.89 \%)$ that decreased to less than half $(26.67 \%$; $p=0.008)$ with the use of intranasal dexmedetomidine (Figure 2).
To measure preoperative anxiety, we used the YPAS scale. It can be used in the pediatric patient and has demonstrated good validity and reliability, both intra-observer and interobserver in numerous clinical trials $[1,10]$.

The intranasal administration of dexmedetomidine was decided to achieve better bioavailability. In pediatric patients, oral transmucosal administration ends up being swallowed, thus losing bioavailability (16\%) compared to intranasal of up to $84 \%[9,14,15]$.

Another of the primary objectives of the study was to compare postoperative delirium in premedicated versus non-premedicated patients as the risk of negative behavior is 3.5 times higher in children who experienced preoperative anxiety, being this an independent predictor $[1,4]$.

Delirium is a dissociated state of consciousness during recovery from general anesthesia with no eye contact with caregivers, inconsolably, disorientation, and involuntary physical activity. It generally begins when the child wakes up from general anesthesia and can take up to 45 minutes to recover [7], so it was decided to measure it within the first hour after surgery. The pediatric anesthesia emergence delirium (PAED) scale of Sikich and Lerman [11], has been used as it is the only validated scale in the pediatric population [9]. A PAED value $>10$ is considered delirium.

The incidence can be up to $80 \%$ depending on different risk factors such as age (older in preschool and school-age), type of anesthesia (use of sevoflurane for a faster awakening) or the type of surgery $[2,8,16]$. We 


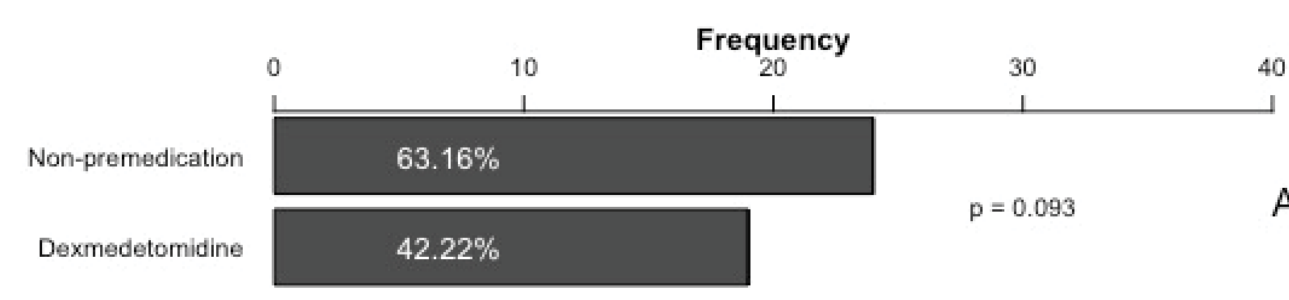

At 15 minutes

Non-premedication

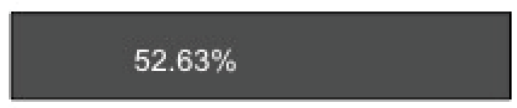

Dexmedetomidine

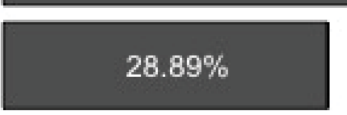

$p=0.048$

At 30 minutes

Non-premedication

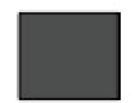

$10.53 \%$

Dexmedetomidine

$22.22 \%$

\section{$p=0.261 \quad$ At one hour}

Non-premedication
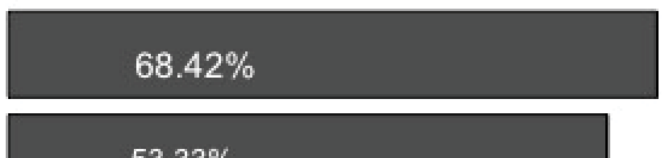

$p=0.24$

At any time post-operatively

Dexmedetomidine

\section{$53.33 \%$}

Figure 3: Postoperative delirium.

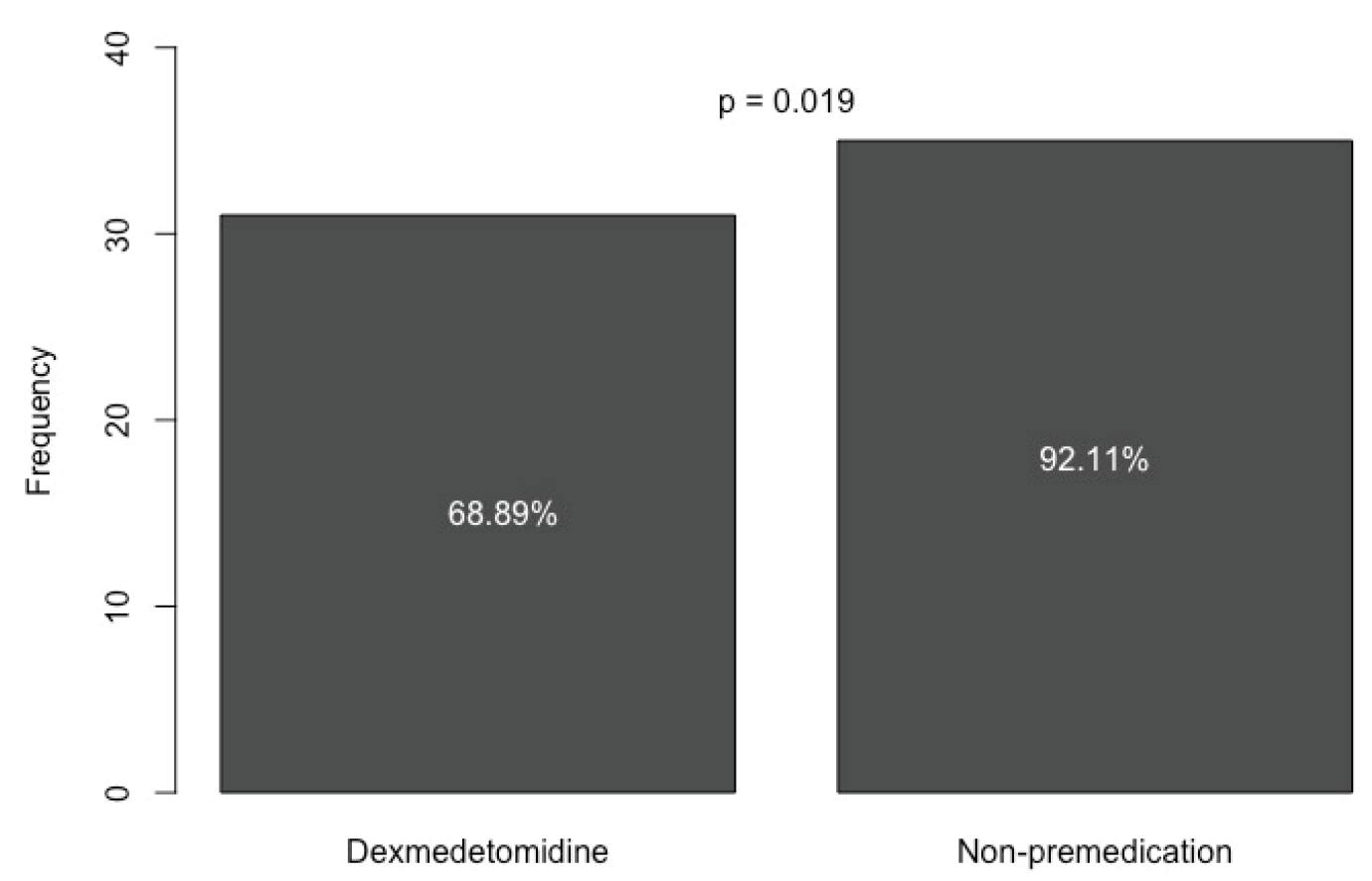

Figure 4: Anxiety or delirium at any time.

observed in our study a high incidence (non-premedicated $68.42 \%$ vs. premedicated $53.33 \%$ ) of postoperative delirium at any time postoperatively. We found no statistically significant differences between the study groups except at 30 minutes after the end of surgery when the incidence of delirium was lower in the group receiving dexmedetomidine. The incidence of postoperative delirium at $15 \mathrm{~min}$ and $30 \mathrm{~min}$ roughly coincides with the 
incidence of preoperative anxiety in non-premedicated, and the incidence of postoperative delirium found in premedicated at 30 minutes and one hour is also similar to the incidence of preoperative anxiety in this group, so the appearance of postoperative delirium in those with preoperative anxiety may be related. However, at 15 minutes the incidence of delirium in premedicated does not coincide with the incidence of preoperative anxiety. Pain does not seem to explain the increase in delirium in the first 15 minutes (OPS =1). One of the possible explanations could be a residual effect of inhaled anesthetics in this period. Also, postoperative delirium at one hour decreases in non-premedicated, possibly related to the residual effect of midazolam that they needed to treat the higher incidence of this complication that they presented at 15 and 30 minutes (Figure 3).

It is especially striking that $92.11 \%$ of non-premedicated children suffered from anxiety or delirium at any time in the perioperative period, decreasing to $68.89 \%$ with dexmedetomidine premedication with a statistically significant difference $(p=0.019)$ (Figure 4).

Several risk factors may have influenced these results. In this case, the comparable age range in both groups, the use of sevoflurane in $100 \%$ of the anesthetics collected, and the type of surgery (tonsillectomy) have been a risk factor for the appearance of delirium. In addition, these OSA patients should not receive premedication with benzodiazepines because they could increase postoperative apnea and PRAEs, so if they aren't premedicated, the risk factors are increased [13].

Inadequate pain control is another possible cause of postoperative delirium [1]. The fact that dexmedetomidine is a potent $\alpha 2$ adrenoreceptor agonist allows it to have analgesic effects, attenuating the stress response without respiratory depression $[6,17]$. Due to these effects, the meta-analysis of $\mathrm{He}$, et al. concludes that the intraoperative use of dexmedetomidine is as effective as the use of opioids to prevent postoperative pain and delirium [6]. In our case, dexmedetomidine was administered only as a premedication and not intra-operatively, therefore the results did not reach this conclusion. In this study, intraoperative fentanyl requirements, postoperative pain or postoperative fentanyl did not show statistically significant differences.

Dexmedetomidine also has sedative effects [7]. Thanks to these effects, it reduces agitation and reduces the consumption of postoperative sedatives in PACU $[6,18]$. Regarding the midazolam needs of this study, in the postoperative period, they were higher in the non-premedicated group but without statistically significant differences.

Regarding PRAEs, it was hypothesized that less use of opioids or perioperative sedatives could decrease their appearance. There was a higher incidence of bron- chospasm, desaturation, and stridor in children without premedication, but these differences were not statistically significant. Therefore, we cannot affirm that premedication with $1 \mu \mathrm{g} / \mathrm{kg}$ dexmedetomidine decreases PRAEs, possibly because this premedication alone failed to decrease the need for opioids and postoperative sedatives.

Although delirium resolves spontaneously, adverse effects such as self-injury or bleeding from the surgical site may occur during its course [19]. In this case, there has been no postoperative bleeding event or need for reoperation.

The most common adverse reactions from dexmedetomidine use are hypotension, hypertension, and bradycardia. These effects have occurred mainly with the use of intravenous dexmedetomidine, especially rapid bolus instead of perfusion [20,21]. In our case, there was no event of hypotension or bradycardia, possibly because the dose was low, and it was administered intranasally.

Although according to this study, with premedication with dexmedetomidine $1 \mu \mathrm{g} / \mathrm{kg}$, anxiety or delirium has been decreased by $23.22 \%$ at any time of the perioperative period, it is necessary to think about how to decrease it even more. Since dexmedetomidine even at high doses of $3 \mu / \mathrm{kg} / \mathrm{h}$ maintains airway tone even in children with OSA [9], it would be feasible to increase the premedication dose and/or add an intraoperative dose or infusion. Further studies are needed to know the safe dose in children and the timing of administration, which results in decreased postoperative delirium, pain, and sedation, and perioperative opioid requirements without producing side effects.

\section{Clinical Implications}

Nowadays, most studies of postoperative agitation in children have been performed with single or continuous intravenous doses of intraoperative or postoperative dexmedetomidine.

Also, the ability of dexmedetomidine to reduce preoperative anxiety has been studied, but although delirium has been described as a negative behavior of preoperative anxiety, there are no studies that relate the decrease in postoperative delirium with a decrease in preoperative anxiety thanks to a correct premedication. The data are limited in defining the dose in premedication or if it would be necessary to add an intraoperative dose of intravenous dexmedetomidine to reduce not only preoperative anxiety but also postoperative delirium and pain.

With this study we want to see if premedicated children have less preoperative anxiety and so then less postoperative delirium without intraoperative intravenous doses. It is hypothesized that intranasal dexmedetomidine premedication decreases the incidence of preope- 
rative anxiety, postoperative delirium, postoperative pain and the need for postoperative sedatives and opioids.

\section{Limitations}

This study has several limitations including non-randomization and the absence of double-blind.

Although it was indicated that premedication is performed 45 minutes before the start of surgery following the protocol approved by the hospital's pharmacy service, the lack of knowledge of the exact time of the start of surgery has led some patients to be transferred to the operating room before or after the effect.

\section{Conclusion}

The incidence of preoperative anxiety in preschool and school-age children who are going to undergo tonsillectomy surgery is very high and this can lead to postoperative delirium in a large number of patients.

Premedication with intranasal dexmedetomidine reduces preoperative anxiety and the incidence of anxiety and delirium at any time in the perioperative period.

At a dose of $1 \mu \mathrm{g} / \mathrm{kg}$, no event of hypotension or bradycardia occurred. There was also no postoperative bleeding event or need for reoperation, nor was the occurrence of PRAEs decreased.

At these doses, there was no decrease in postoperative pain or less need for intraoperative or postoperative opioids or postoperative sedatives.

More studies are needed to establish effective and safe doses in pediatric patients of both preoperative and intraoperative dexmedetomidine.

\section{Acknowledgments}

Patricia Catalán Escudero: The conception and design of the study, patient recruitment, data collection, interpretation of data, and drafting the article.

Adolfo Ramos Luengo: Study design and data analysis and interpretation of data, revising it critically for important content.

Eva Delgado Encinas: Data collection and revising the article critically.

Ernesto Martínez García: Patient recruitment, revising it critically for important content.

\section{Conflicts of Interest}

The authors declare no competing interests.

\section{References}

1. Banchs RJ, Lerman J (2014) Preoperative anxiety management, emergence delirium, and postoperative behavior. Anesthesiol Clin 32: 1-23.

2. Shi M, Miao S, Gu T, Wang D, Zhang H, et al. (2019) Dexmedetomidine for the prevention of emergence delirium and postoperative behavioral changes in pediatric patients with sevoflurane anesthesia: A double-blind, randomized trial. Drug Des Devel Ther 13: 897-905.

3. Amorim MA, Govêia CS, Magalhães E, Ladeira LC, Moreira LG, et al. (2017) Effect of dexmedetomidine in children undergoing general anesthesia with sevoflurane: A meta-analysis. Rev Bras Anestesiol 67: 193-198.

4. Fitz Simons J, Bonanno LS, Pierce S, Badeaux J (2017) Effectiveness of preoperative intranasal dexmedetomidine, compared with oral midazolam, for the prevention of emergence delirium in the pediatric patient undergoing general anesthesia: A systematic review. JBI Database System Rev Implement Rep 15: 1934-1951.

5. Yuen VM, Bailey CR (2018) Premedication in children: does taste matter?. Anaesthesia 73: 1453-1456.

6. He XY, Cao JP, Shi XY, Zhang H (2013) Dexmedetomidine versus morphine or fentanyl in the management of children after tonsillectomy and adenoidectomy: A meta-analysis of randomized controlled trials. Ann Otol Rhinol Laryngol 122: 114-120.

7. Plambech MZ, Afshari A (2015) Dexmedetomidine in the pediatric population: A review. Minerva Anestesiol 81: 320332.

8. Dahmani S, Delivet H, Hilly J (2014) Emergence delirium in children: An update. Curr Opin Anaesthesiol 27: 309-315.

9. Mahmoud M, Mason KP (2015) Dexmedetomidine: Review, update, and future considerations of paediatric perioperative and periprocedural applications and limitations. $\mathrm{Br}$ J Anaesth 115: 171-182.

10. Kain ZN, Mayes LC, Cicchetti DV, Bagnall AL, Finley JD, et al. (1997) The yale preoperative anxiety scale: How does it compare with a "gold standard"? Anesth Analg 85: 783788.

11. Sikich N, Lerman J (2004) Development and psychometric evaluation of the pediatric anesthesia emergence delirium scale. Anesthesiology 100: 1138-1145.

12. Cao JL, Pei YP, Wei JQ, Zhang YY (2016) Effects of intraoperative dexmedetomidine with intravenous anesthesia on postoperative emergence agitation/delirium in pediatric patients undergoing tonsillectomy with or without adenoidectomy : A CONSORT-prospective, randomized, controlled clinical trial. Medicine (Baltimore) 95: e5566.

13. Guler G, Akin A, Tosun Z, Ors S, Esmaoglu A, et al. (2005) Single-dose dexmedetomidine reduces agitation and provides smooth extubation after pediatric adenotonsillectomy. Paediatr Anaesth 15: 762-766.

14. Z Serhan Cimen, Ayse Hanci, G Ulufer Sivrikaya, Leyla T Kilinc, Melahat K Erol (2013) Comparison of buccal and nasal dexmedetomidine premedication for pediatric patients. Pediatric anesthesia 23: 134-138.

15. Miller JW, Balyan R, Dong M, Mahmoud M, Lam JE, et al. (2018) Does intranasal dexmedetomidine provide adequate plasma concentrations for sedation in children: A pharmacokinetic study. Br J Anaesth 120: 1056-1065.

16. Moore AD, Anghelescu DL (2017) Emergence delirium in pediatric anesthesia. Paediatr Drugs 19: 11-20.

17. Olutoye OA, Glover CD, Diefenderfer JW, McGilberry M, Wyatt MM, et al. (2010) The effect of intraoperative dexmedetomidine on postoperative analgesia and sedation in pediatric patients undergoing tonsillectomy and adenoidectomy. Anesth Analg 111: 490-495.

18. Bellon M, Le Bot A, Michelet D, Hilly J, Maesani M, et al. (2016) Efficacy of intraoperative dexmedetomidine com- 
pared with placebo for postoperative pain management: $A$ meta-analysis of published studies. Pain Ther 5: 63-80.

19. Cho EA, Cha YB, Shim JG, Ahn JH, Lee SH, et al. (2020) Comparison of single minimum dose administration of dexmedetomidine and midazolam for prevention of emergence delirium in children: A randomized controlled trial. J Anesth 34: 59-65.
20. Peng K, Wu SR, Ji FH, Li J (2014) Premedication with dexmedetomidine in pediatric patients: A systematic review and meta-analysis. Clinics (Sao Paulo) 69: 777-786.

21. Pérez-Guillé MG, Toledo-López A, Rivera-Espinosa L, Alemon-Medina R, Murata C, et al. (2018) population pharmacokinetics and pharmacodynamics of dexmedetomidine in children undergoing ambulatory surgery. Anesth Analg 127: 716-723. 